\title{
ultramicroscopy
}

ELSEVIER

Ultramicroscopy 77 (1999) 129-134

\section{Improvements in the reconstruction of in-line holograms by energy sampling and tomography: II}

\author{
Mark R.A. Shegelski*, Thomas Clark, Matthew Reid, Silas Faltus \\ Department of Physics, University of Northern British Columbia, 3333 University Way, Prince George, BC, Canada V2N $4 Z 9$
}

Received 28 August 1998; received in revised form 2 February 1999

\begin{abstract}
Depth resolution in the reconstruction of in-line holograms taken with the low-energy electron point source (LEEPS) microscope is improved by employing, on the basis of a Kirchhoff-Helmholtz-type reconstruction integral, (i) a tomographic approach, sampling several screen positions, and (ii) a weighted energy averaging. The improved reconstructions exhibit atomic resolution both laterally and in depth. We use an analytical approach to average over a selected range of screen positions to obtain such improved reconstructions. We investigate how the improvement depends on the range of screen positions and on screen size. (C) 1999 Elsevier Science B.V. All rights reserved.
\end{abstract}

PACS: $61.16 . \mathrm{B} ; 61.14 . \mathrm{N}$

Keywords: Electron microscopy; Reconstruction; Tomography

\section{Introduction}

In a previous work [1], we obtained improved reconstructions of in-line holograms for low-energy electron point source (LEEPS) microscopy. Our principal result was to altogether eliminate spurious peaks in the source-object direction by sampling over several screen positions in conjunction with a weighted energy sampling. The idea behind sampling over screen positions is that for a spherical screen completely surrounding the (perfect point) source, i.e. recording the complete hologram, reconstruction yields resolution of the order of the

\footnotetext{
* Corresponding author. Tel.: + 1-250-960-6663; fax: + 1250-960-5545.

E-mail address: mras@unbc.ca (M.R.A. Shegelski)
}

wavelength in all directions. This is obviously not feasible experimentally and so far one has recorded the hologram on a flat screen of limited size positioned perpendicular to the optical axis. We showed that by rotating a flat screen through a few angles away from the optical axis, one records enough information to improve the depth resolution in reconstruction to equal the lateral resolution. (See the geometry in Fig. $1 ; \eta$ is the angle through which the screen is rotated from the optical axis.) Note that moving the screen around the source is equivalent to moving the object off axis for a fixed screen so that it is illuminated from different directions. This method is thus akin to tomography. An additional weighted energy average, over a range easily accessible in the LEEPS microscope, was shown to then remove all spurious peaks in reconstruction. 


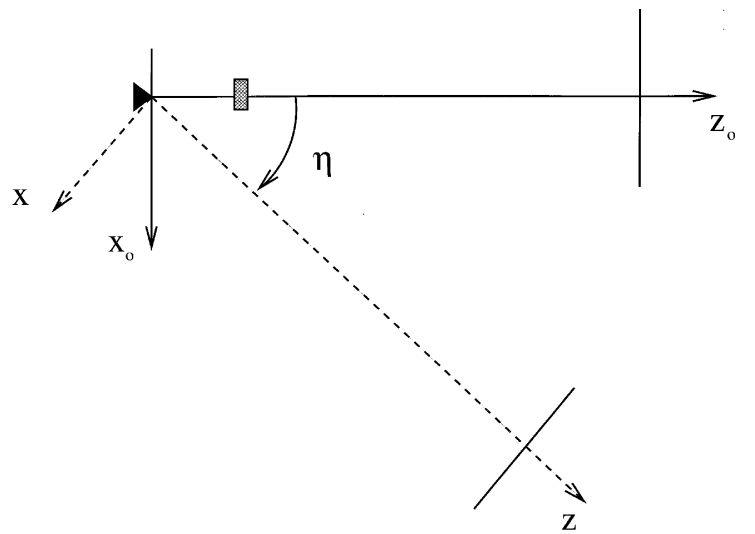

Fig. 1. Schematics of the coordinate systems used in the simulations.

The results of our previous work are summarized below:

(1) Averaging over screen positions significantly improves the reconstruction of atomic positions, especially in the source-object direction (the $z_{0^{-}}$ direction), without loss of sharpness perpendicular to the source-object direction (the $x_{0}-y_{0}$ plane).

(2) A weighted averaging over different electron energies also significantly improves the reconstruction of atomic positions, especially in the $z_{0}$-direction, again without loss of sharpness in the $x_{0}-y_{0}$ plane; the improvements are in harmony with screen averaging.

(3) Screen averaging over a finite number of screen positions (typically 3-5) gives reconstructions that are just as good as averaging over a continuum of screen positions.

(4) Energy averaging over a finite number of discrete energies (typically 10) gives reconstructions that are just as good as averaging over a continuum of energies.

(5) Energy averaging in the range $80-200 \mathrm{eV}$ gives much better reconstruction than the range $0-200 \mathrm{eV}$.

(6) Discrete screen position sampling combined with discrete energy sampling removes all spurious peaks for all the atomic clusters we have investigated to date: sharp peaks result at atom positions and only at atom positions.

The primary purpose of this paper is to exploit an analytical formula for averaging over screen positions with the intention of investigating how the quality of the improved reconstructions depends on the range of screen positions and on the size of the screen. We first present the analytical formula for screen position averaged reconstruction. Use of this analytical result greatly facilitates our theoretical investigation into the dependence of the quality of reconstructions on the range of screen positions and the screen size. We will show that, even for a fairly small screen size and a relatively small range of screen positions, significant improvements in reconstruction are still obtained. We show that, even when using a small screen and averaging over a small range, atomic resolution is achieved both laterally and in depth, when screen averaging and energy averaging are combined. These findings were reported in our previous paper. In this paper, the emphasis is on the analytical approach to facilitate investigations into screen size and range of screen positions.

Our methods of screen position and energy averaging are described in detail in Ref. [1]. References to related works are also given in Ref. [1].

\section{Analytical formula for reconstruction}

The role of reconstruction is to obtain the threedimensional structure of the object from the twodimensional hologram on the screen. This can be achieved via a Kirchhoff-Helmholtz transform [2]

$K(\boldsymbol{r})=\int_{S} \mathrm{~d}^{2} \xi I(\xi) \exp (\mathrm{i} k \xi \cdot \boldsymbol{r} / \xi)$,

where the integration extends over the two-dimensional surface of the screen with coordinates $\xi=(X, Y, L)$ where $L$ is the distance from the source to the (center of the) screen, $k=2 \pi / \lambda$ is the wave vector of the electrons, and $I(\xi)$ is the contrast image on the screen obtained by subtracting the images with and without the object present. The function $K(\boldsymbol{r})$ is significantly structured and different from zero only in the spatial region occupied by the object. By reconstructing the wave front $K(\boldsymbol{r})$ on a number of planes at various distances from the source in the vicinity of the object, a three-dimensional image can be built up. 
To further explore improvements in the reconstruction procedure we will make use of simulated, rather than experimental, LEEPS images to avoid limitations and possible artifacts of the experiment. Kreuzer et al. [2] have developed a scattering theory for the simulation of images in which the object is represented by its atomic constituents. The integral in Eq. (1) was evaluated analytically for a circular screen (i.e. a disk) in Ref. [2]. Our starting point is Eq. (2) for $K(\boldsymbol{r})$ in Ref. [1] (see also Eqs. (3)-(6) in Ref. [1]). In this paper we focus on $s$-wave scattering. Consequently, the expressions are considerably simpler than those in Ref. [1]. We have compared our analytical results for $s$-wave scattering only (to be presented in this paper) with those obtained numerically via the methods we used in Ref. [1]; we find that taking $s$-wave scattering only is a reasonable approximation.

Averaging over a continuum of screen positions gives the following analytical formula for $s$-wave scattering:

$$
\begin{aligned}
\langle K(\boldsymbol{r})\rangle_{s}= & \sum_{i} \sum_{n \geqslant 0} \tau_{0} F_{0}\left(\boldsymbol{r}_{i}\right)\left(\frac{2 l+1}{4 \pi}\right)^{1 / 2} \\
& \times i^{n} a_{n} I_{n}\left(\left|\boldsymbol{r}-\boldsymbol{r}_{i}\right| ; \eta_{0}\right) j_{n}\left(k\left|\boldsymbol{r}-\boldsymbol{r}_{i}\right|\right),
\end{aligned}
$$

where

$I_{n}\left(\left|\boldsymbol{r}-\boldsymbol{r}_{i}\right| ; \eta_{0}\right) \equiv \frac{1}{2 \eta_{0}} \int_{-\eta_{0}}^{\eta_{0}} \mathrm{~d} \eta P_{n}\left[\frac{z(\eta)-z_{i}(\eta)}{\left|\boldsymbol{r}-\boldsymbol{r}_{i}\right|}\right]$,

$z(\eta)-z_{i}(\eta)=\left(z_{0}-z_{i, 0}\right) \cos (\eta)+\left(x_{0}-x_{i, 0}\right) \sin (\eta)$,

$\tau_{0}=k^{-1} \sin \left(\delta_{0}\right) \exp \left(\mathrm{i} \delta_{0}\right)$,

$F_{0}\left(\boldsymbol{r}_{i}\right) \approx(4 \pi)^{1 / 2} r_{i}^{-1} \mathrm{e}^{\mathrm{i} k r_{i}}$,

and

$a_{n}=\frac{2 n+1}{2} \int_{\cos \theta_{\max }}^{1} \mathrm{~d} t P_{n}(t)$.

Here the $P_{n}$ are Legendre polynomials, the $j_{n}\left[k\left|\boldsymbol{r}-\boldsymbol{r}_{i}\right|\right]$ are spherical Bessel functions, and $\delta_{0}(k)$ is the phase shift at wave vector $k$. The $x-, y$-, and $z$-axis have their origin at the point source, with the $z$-axis passing through the center of the screen; the angle $\theta_{\max }$ is the angle from the center of the screen to its edge. Note that for $\theta_{\max }=\pi$ we have $a_{n}=\delta_{0, n}$.
We emphasize that the integration in (3), for any $n$, is readily carried out analytically. Consequently, Eq. (2) provides us with an analytical formula which facilities our theoretical investigation.

\section{Results and discussion}

We present results for two different clusters, three screen sizes, and for averages over the ranges $|\eta| \leqslant \pi / 4,|\eta| \leqslant \pi / 8$, and $|\eta| \leqslant \pi / 16$. The smallest screen has a diameter of $7 \mathrm{~cm}$, the medium-sized screen has twice the area of the smallest, and the largest has 4 times the area of the $7 \mathrm{~cm}$ screen. The source-object distance is $1000 \AA$, and the sourcescreen distance is $10 \mathrm{~cm}$.

We begin with a $5 \times 5 \mid 4 \times 4$ double layer of carbon atoms. The layers are in $x_{0}-y_{0}$ planes with the $5 \times 5$ layer a distance $d=1000 \AA$ from the point source, arranged on a square lattice with spacing $a=2.5 \AA$, and with the $4 \times 4$ layer at $d=1004.5 \AA$, mimicking an FCC lattice. Fig. 2 shows the reconstructions $K(\boldsymbol{r})$ along the $z_{0}$-axis for the largest of the screens. The "unaveraged curve" was obtained from the unaveraged version of Eq. (2) (see Ref. [2]) and exhibits essentially the same form as the curves in Ref. [2]. The "screen averaged curves" were obtained using the analytical formula (2). The principal consequence of the averaging is more sharply resolved peaks. In particular, note the deepening of the minima between the two principal peaks.

Combining the weighted energy averaging method (see Ref. [1]) with screen averaging over the range $|\eta| \leqslant \pi / 8$ removes the spurious peaks, resulting in a single, sharp peak at the atomic site, also shown in Fig. 2.

Fig. 3 again shows $z_{0}$-cuts for the $5 \times 5 \mid 4 \times 4$ cluster, with the same screen averagings and energy averaging, but for a screen with half the area of the largest screen. The same conclusions apply for this smaller screen as did for the larger screen. The importance of this is that reconstructions of the same calibre can be done with a smaller screen. Given that 40 other atoms are close to, but not on, the $z_{0}$-axis, the sharpness of the single peak is a significant improvement over the unaveraged curve.

Figs. 4 and 5 are the analogues of Figs. 2 and 3; these reconstructions are for a 4-layer carbon 


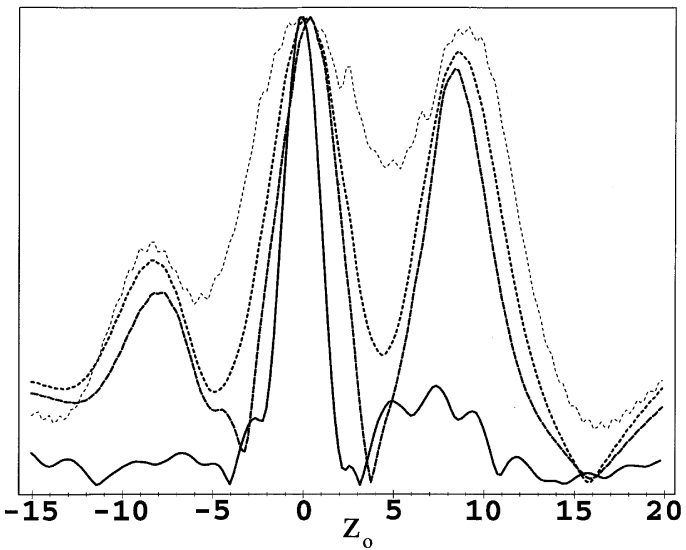

Fig. 2. The unaveraged (light, short dashed line), and screen averaged reconstructions, with $|\eta| \leqslant \pi / 8$ (short dashed line), and $|\eta| \leqslant \pi / 4$ (long dashed line) for a $5 \times 5 \mid 4 \times 4$ carbon cluster. Also shown is the screen and energy averaged reconstruction (solid line) (with $|\eta| \leqslant \pi / 8$ ). The cut is along the $z_{0}$ direction through the central atom of the $5 \times 5$ layer; $z_{0}=0$ corresponds to the $5 \times 5$ layer (i.e. $1000 \AA$ from the source); the units of distance are $\AA$. These curves were obtained using the largest screen size; see text for details.

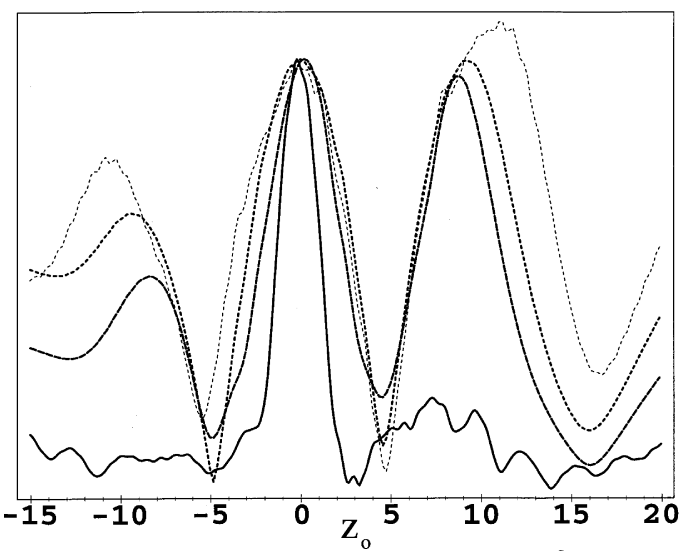

Fig. 3. As in Fig. 2 but for the mid-sized screen.

cluster with the structure $5 \times 5|4 \times 4| 5 \times 5 \mid 4 \times 4$. Again, the screen averaging reveals peaks at the two atom locations, and the combined screen and energy averaging curve shown in Fig. 4 shows sharp peaks at the two atomic sites and only at these sites. Of note is that 80 other atoms are close to but not on the $z_{0}$-axis.

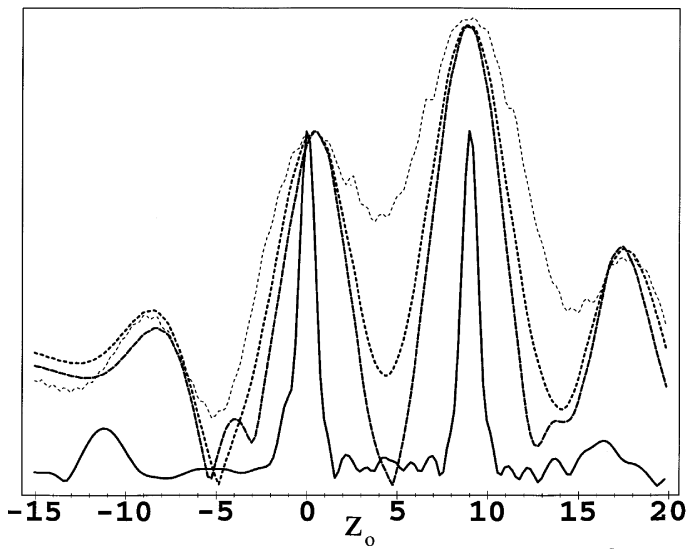

Fig. 4. As in Fig. 2, again using the large screen, but for a $5 \times 5|4 \times 4| 5 \times 5 \mid 4 \times 4$ carbon cluster; $z_{0}=0$ and $z_{0}=9$ correspond to the $5 \times 5$ layers.

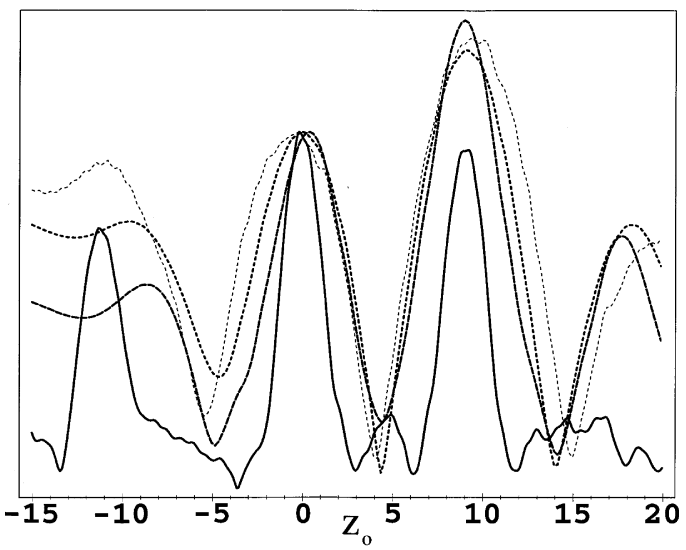

Fig. 5. As in Fig. 3, again using the mid-sized screen, but for a $5 \times 5|4 \times 4| 5 \times 5 \mid 4 \times 4$ carbon cluster.

Fig. 6 shows $z_{0}$-cuts for the combined averaging, using the mid-sized screen, with different ranges of screen positions. The spurious peak diminishes as the range increases from $|\eta| \leqslant \pi / 8$ to $|\eta| \leqslant \pi / 4$.

Figs. $7-10$ give results obtained using the smallest screen: $7 \mathrm{~cm}$ in diameter. Unaveraged, screen averaged, and screen and energy averaged curves are presented. Ranges of $|\eta| \leqslant \pi / 4,|\eta| \leqslant \pi / 8$, and $|\eta| \leqslant \pi / 16$ were examined.

Figs. 7 and 9 are for the $5 \times 5 \mid 4 \times 4$ cluster, Figs. 8 and 10 for the $5 \times 5|4 \times 4| 5 \times 5 \mid 4 \times 4$ cluster; $z$-cuts are shown in Figs. 7 and 8; Figs. 9 and 10 are $x$-cuts. 


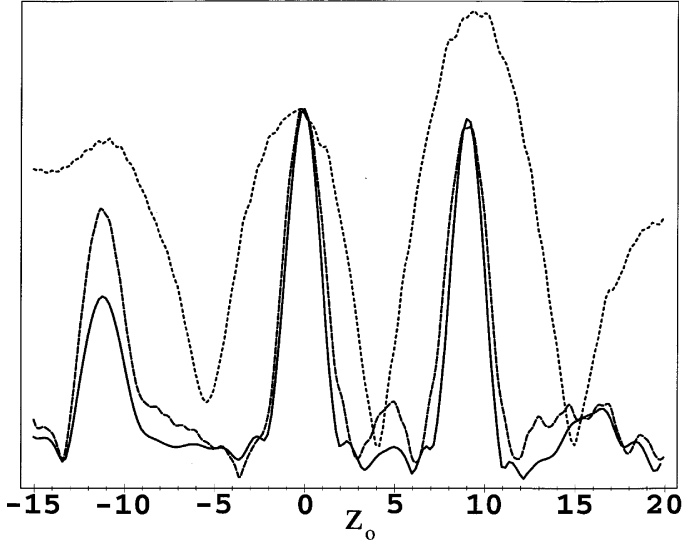

Fig. 6. Screen and energy averaged reconstructions for the $5 \times 5|4 \times 4| 5 \times 5 \mid 4 \times 4$ carbon cluster, with $|\eta| \leqslant \pi / 8$ (dashed line), and $|\eta| \leqslant \pi / 4$ (solid line). Also shown is the unaveraged curve (short dashed line). The cut is along the $z_{0}$ direction through the middle of the $5 \times 5$ layers. The mid-sized screen was used.

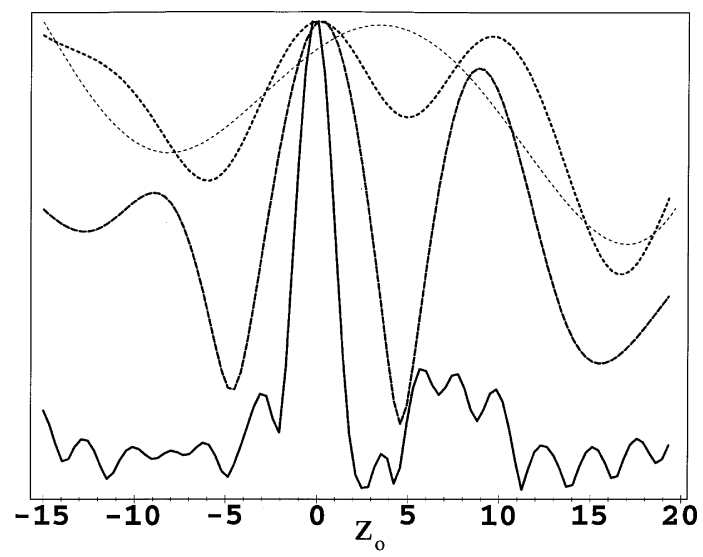

Fig. 7. The unaveraged reconstruction (light, short dashed line), the screen averaged reconstructions, with $|\eta| \leqslant \pi / 16$ (short dashed line), and with $|\eta| \leqslant \pi / 8$ (long dashed line), and the screen and energy averaged reconstruction, with $|\eta| \leqslant \pi / 8$ (solid line), for a $5 \times 5 \mid 4 \times 4$ carbon cluster, using the smallest screen $(7 \mathrm{~cm})$. The cut is as in Fig. 2, along the $z_{0}$ direction.

From Figs. 7 and 8 we conclude that, for the $7 \mathrm{~cm}$ screen, atomic resolution in depth results for the $5 \times 5 \mid 4 \times 4$ cluster provided the range $|\eta| \leqslant \pi / 8$ is combined with the energy averaging method (Fig. 7). For the $5 \times 5|4 \times 4| 5 \times 5 \mid 4 \times 4$ cluster, the angular range must be increased to $|\eta| \leqslant \pi / 4$ to significantly diminish the spurious peak (Fig. 8). In Figs. 9

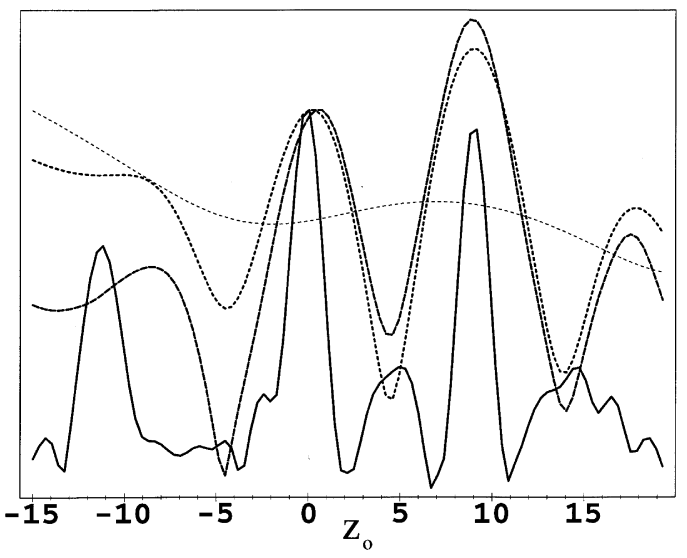

Fig. 8. The unaveraged reconstruction (light, short dashed line), the screen averaged reconstructions, with $|\eta| \leqslant \pi / 8$ (short dashed line), and with $|\eta| \leqslant \pi / 4$ (long dashed line), and the screen and energy averaged reconstruction, with $|\eta| \leqslant \pi / 4$ (solid line), for a $5 \times 5|4 \times 4| 5 \times 5 \mid 4 \times 4$ carbon cluster, using the smallest screen $(7 \mathrm{~cm})$. The cut is along the $z_{0}$-direction through the central atoms of the $5 \times 5$ layers.

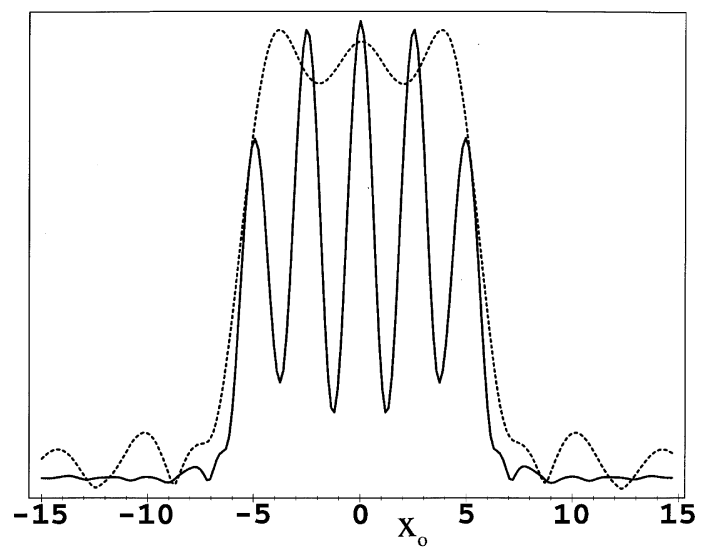

Fig. 9. The unaveraged (dashed line), and the screen averaged (with $|\eta| \leqslant \pi / 8$ ) (solid line) reconstructions for the $5 \times 5 \mid 4 \times 4$ carbon cluster for the smallest $(7 \mathrm{~cm})$ screen. The cut is along the $x_{0}$-direction through the middle of the $5 \times 5$ layers.

and 10 , we see vast improvement in the lateral reconstruction with screen averaging only. Atomic resolution is obtained laterally for both clusters provided a range of $|\eta| \leqslant \pi / 8$ of screen positions is used (the smaller range $|\eta| \leqslant \pi / 16$, e.g., fails to give sufficient resolution). 


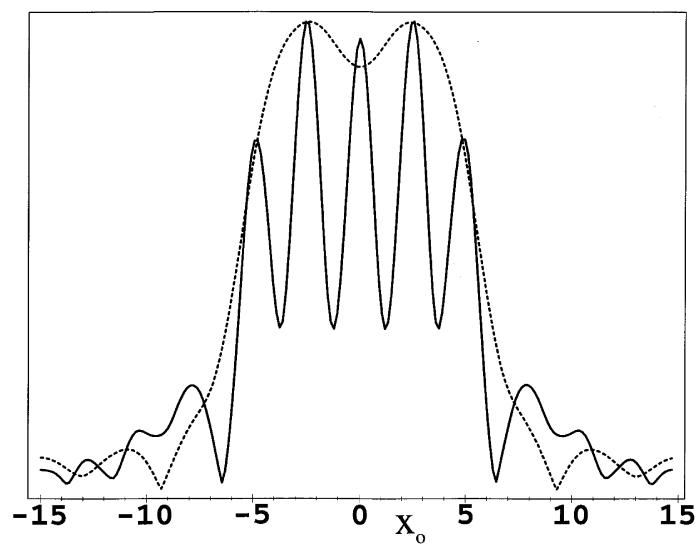

Fig. 10. As in Fig. 9 but for a $5 \times 5|4 \times 4| 5 \times 5 \mid 4 \times 4$ carbon cluster.

The results we have given were readily generated through the use of an analytical formula, obtained by integrating over a continuum of $\eta$-values. This simplifies the theoretical analysis. However, as discussed in detail in our previous work [1], the same results can be obtained with a finite number of screen positions.

\section{Summary}

The principal results of this paper are as follows.

(1) We have presented an analytical formula for averaging over screen positions, with the (center of the) screen kept a fixed distance from the source and kept perpendicular to the line from the source to the (center of the) screen; the screen positions are given by the angle $\eta$ through which the screen is rotated from the optical axis. The analytical formula facilitates our theoretical inquiry into improvements in the reconstructions of in-line holograms.

(2) Averaging over screen positions significantly improves the reconstruction of atomic posi- tions, especially in the $z_{0}$-direction, without loss of sharpness in the $x_{0}-y_{0}$ plane; use of the analytical formula for screen averaging shows that improved reconstructions result even when the range of averaging is restricted to $|\eta| \leqslant \pi / 8$, or even $|\eta| \leqslant \pi / 16$, and when the screen size is relatively small.

(3) The combination of averaging over screen positions with a weighted energy averaging removes almost all spurious peaks for the atomic clusters we have investigated to date: sharp peaks result at atom positions and only at atom positions. The high quality of the reconstructions is still obtained with a smaller screen and when the averaging over screen positions is restricted to a smaller range, e.g. $|\eta| \leqslant \pi / 8$, or even $|\eta| \leqslant \pi / 16$.

Further work along the lines of this paper is needed. For example, the development of a method requiring either only tomographic averaging, or only energy averaging, with reconstructions of the calibre illustrated in this paper, would be highly desirable. An investigation into reconstructions obtained along these lines is currently underway.

\section{Acknowledgements}

This work was supported by the Natural Sciences and Engineering Research Council of Canada (NSERC). One of us (M.R.) acknowledges receipt of an undergraduate NSERC research award. We thank Prof. H.J. Kreuzer for useful conversations and constructive comments.

\section{References}

[1] M.R.A. Shegelski, T. Clark, S. Faltus, H.J. Kreuzer, Ultramicroscopy 74 (1998) 169.

[2] H.J. Kreuzer, K. Nakamura, A. Wierzbicki, H.-W. Fink, H. Schmid, Ultramicroscopy 45 (1992) 381. 